\title{
A monoclonal antibody raised against bacterially expressed MPV17 sequences shows peroxisomal, endosomal and lysosomal localisation in U2OS cells
}

Hans Weiher ${ }^{1,2^{*}}$, Haymo Pircher ${ }^{3}$, Pidder Jansen-Dürr ${ }^{3}$, Silke Hegenbarth ${ }^{4}$, Percy Knolle ${ }^{4}$, Silke Grunau ${ }^{5}$, Miia Vapola ${ }^{5}$, J. Kalervo Hiltunen ${ }^{5}$, Ralf M. Zwacka ${ }^{6}$, Elmon Schmelzer ${ }^{7}$, Kerstin Reumann ${ }^{1}$ and Hans Will ${ }^{1}$

\begin{abstract}
Recessive mutations in the MPV17 gene cause mitochondrial DNA depletion syndrome, a fatal infantile genetic liver disease in humans. Loss of function in mice leads to glomerulosclerosis and sensineural deafness accompanied with mitochondrial DNA depletion. Mutations in the yeast homolog Sym 1, and in the zebra fish homolog tra cause interesting, but not obviously related phenotypes, although the human gene can complement the yeast Sym 1 mutation. The MPV17 protein is a hydrophobic membrane protein of 176 amino acids and unknown function. Initially localised in murine peroxisomes, it was later reported to be a mitochondrial inner membrane protein in humans and in yeast. To resolve this contradiction we tested two new mouse monoclonal antibodies directed against the human MPV17 protein in Western blots and immunohistochemistry on human U2OS cells. One of these monoclonal antibodies showed specific reactivity to a protein of $20 \mathrm{kD}$ absent in MPV17 negative mouse cells. Immunofluorescence studies revealed colocalisation with peroxisomal, endosomal and lysosomal markers, but not with mitochondria. This data reveal a novel connection between a possible peroxisomal/endosomal/lysosomal function and mitochondrial DNA depletion.
\end{abstract}

Keywords: MPV17 monoclonal antibody, Mitochondrial DNA depletion syndrome, Lysosomes, Endosomes, Mitochondria, Peroxisomes

\section{Findings}

\section{Background}

Mutations in the human MPV17 gene have been firstly discovered to be causal for the lethal liver disease Mitochondrial DNA Depletion Syndrome (MDDS) by Spinazzola et al. in 2006 [21]. Since then various additional mutations within this gene were described to cause the syndrome $[5,8,23]$. In contrast to other proteins of which gene mutations cause MDDS such as POLG, TK2, or DGOUK [4], this protein is not obviously involved in replication or in nucleic acid metabolism, but appears

\footnotetext{
*Correspondence: hans.weiher@fh-brs.de; hans.weiher@h-brs.de

2 Bonn-Rhein-Sieg University, von Liebig Strasse 20, 53359 Rheinbach,

Germany

Full list of author information is available at the end of the article
}

to be a membrane protein of unknown molecular function $[21,25,26] . M P V 17$ gene knockout in mice has been described earlier as causal of glomerulosclerosis [2, 14, 25] and inner ear disease [11], reminiscent of chemical damage by Adriamycin [15]. Although these mice also showed mitochondrial DNA depletion, they displayed no major liver phenotype [24]. A functional link between the PRKDC repair protein, the mouse kidney phenotype as well as the mitochondrial depletion phenotype has been established by Papeta et al. [15]. Human MPV17 protein expression can rescue the phenotype in transgenic mice, negative for murine MPV17 [20]. A functional homolog of the MPV17 protein was identified in yeast (Sacharomyces cerevisiae) and the human gene can rescue the yeast phenotype in Sym1 negative yeast as well 
[22]. In addition, a mutant in the zebra fish (Dario rerio) MPV17 homolog tra has been described [12]. The yeast and fish mutant phenotypes appear remarkably different from the mutant phenotypes in mammals: Sym 1 negative yeast fails to grow at elevated temperature in ethanol [22], while tra negative fish are viable but show a transparent appearance [12]. Finally, MPV17 is a member of a protein family, including MPV17 like proteins $[6,19]$ and the peroxisomal membrane protein PXMP22 [7, 18]. The hypothesis that MPV17 protein might constitute a channel allowing-in vertebrates-nucleotides or-in yeast-metabolic intermediates to pass though internal membranes was recently reviewed [13].

The publication describing the causative role of MPV17 mutations in the human liver disease MDDS included an analysis of the intracellular localisation of the MPV17 protein in human cells based mainly on studies of transfected cells over-expressing a c-terminally tagged recombinant protein, it was stated that this protein localised to the inner membrane of mitochondria [21]. Supporting this notion, it has been established that the yeast homolog of MPV17, SYM1, indeed localises to this cell compartment [17, 22]. An earlier study [26], however, had localised the MPV17 gene product to peroxisomes, again based on immunofluorescence data and supported by the similarity of the protein to the bona-fide peroxisomal membrane protein PXMP22 [7].

To clarify this contradiction we here present co-localisation studies using novel anti-human MPV17 monoclonal mouse antibodies.

\section{Methods}

\section{Cell lines and tissue culture}

Human osteosarcoma cells (U2OS) cells were originally obtain from the American tissue culture collection $\left(\right.$ ATCC $^{\circledR}$ HTB- $96^{\mathrm{TM}}$ ), kept at the Heinrich Pette Institut for several years and used in [10]. Primary murine embryonic fibroblasts (MEF) were produced from day 11 MPV17 +/+ and MPV17 -/- embryos respectively according to Zwacka et al. [26]. U2OS and mouse MEF cells were cultured under standard conditions. Stable transformants were selected using $1 \mathrm{mg} / \mathrm{ml} \mathrm{G} 418$ (Life Technologies). Hybridomas were generated as described [26], and clones were grown in RMPI media including $10-20 \%$ FCS. The clones were monitored for IgG production by immunoblotting using anti-IgG antibodies and the ECL System (Pierce) for detection. Antibodies were purified using a Protein G Sepharose column (Life Technologies) according to the manufacturer's instructions.

\section{Antibodies and organelle detection}

Commercial primary antibodies against MPV17 were: mouse monoclonal anti-human MPV17 antibody
(60103-1-Ig, Proteintech), rabbit anti-human MPV17 polyclonal antibody (10310-1-AP, Proteintech); rabbit anti-human MPV17 polyclonal antibody (ab93374, Abcam); goat anti-human MPV17 polyclonal antibody (sc-109551, Santa Cruz), rabbit anti-MPV17 C-terminal region polyclonal antibody ARP73712-P050 Insight Biotechnology), rabbit anti-MPV17 N-terminal region polyclonal antibody AP8749a-ev-AB, BioCat).

Further primary antibodies used were: mouse polyclonal anti-human catalase antibody (Abcam ab88650); mouse monoclonal anti-complex IV mitochondrial subunit I (Invitrogen 459600); rabbit polyclonal anti-human PMP70 antibody (gift from Wilhelm Just, University of Heidelberg); rabbit anti-human Rab 7 antibody (Sigma R4779); goat anti- human cathepsin D antibody (Santa Cruz sc 6486); rabbit anti-human EEA1 antibody (NovusBiologicals, NB-300-502); goat anti-human LAMP1 antibody (Santa Cruz, sc 8098); mouse monoclonal anti-flag antibody (Sigma, M2, F3165), mouse monoclonal antihuman beta-actin antibody (Sigma, AC74, A5316).

Primary monoclonal Antibodies 5D2 and 6F5 were isolated from mass cultures and purified over protein G Sepharose (Life Technologies). A Rabbit polyclonal anti-human PMP70 antibody was a gift from Wilhelm Just, University of Heidelberg. Further primary antibodies used were: rabbit anti-human Rab 7 antibody (Sigma R4779); goat anti-human cathepsin D antibody (Santa Cruz sc 6486); rabbit anti-human EEA1 antibody (NovusBiologicals, NB-300-502); goat anti-human LAMP1 antibody (Santa Cruz, sc 8098); mouse monoclonal antihuman beta-actin antibody (Sigma, AC74, A5316).

Secondary antibodies: HRP labeled anti-mouse IgG antibody (Pierce); Alexa Fluor 555 and Alexa Fluor 488 with appropriate anti-mouse, anti-rabbit, and anti-goat specificity were from Life Technologies. For mitochondrial staining, MitoTracker Red 7510 was employed according to the manufacturer's instructions.

\section{Cell extracts and immunoblotting}

Cell extracts were prepared and the immunoblotting was performed as described by Kinkley et al. [10] and Pircher et al. [16].

\section{Immunofluorescence analysis}

Immunofluorescence analysis was performed essentially as described by Kinkley et al. [10]. In brief, cells were grown on cover slips, fixed with $4 \%$ paraformaldehyde in PBS (10 min at room temperature), washed twice with PBS, permeabilized with $0.5 \%$ Triton X-100 in PBS (10 min at room temperature), washed three times in PBS and then blocked for $1 \mathrm{~h}$ (at room temperature) or overnight $\left(4{ }^{\circ} \mathrm{C}\right)$ with $3 \% \mathrm{BSA}$ in PBS. Cover slips were then incubated in $200 \mu \mathrm{l}$ of primary antibodies, diluted 1/200 
in PBS, for $1 \mathrm{~h}$, washed three times with PBS and then incubated with the appropriate secondary antibodies for $1 \mathrm{~h}$. The cover slips were then washed three more times in PBS and mounted on slides using Mowiol (Calbiochem). The cells were analyzed by indirect immunofluorescence microscopy using a Zeiss LSM confocal microscope or a MicroRadiance confocal scanning system (Bio-Rad) in combination with a Zeiss Axiophot microscope (Fig. 1). Colocalisation analysis was performed with Fiji (Image J).

\section{Plasmid constructs}

An MPV17 expression construct (SC118652) was from origene. For details see: www.origene.com/cdna/.

\section{Results}

Commercially available anti-human MPV17 antibodies from different sources (Abcam ab93374; Proteintech 10310-1-AP, 60310-1-Ig; Santa Cruz Biotechnology SC109551; Insight Biotechnology ARP73712-P050; BioCat AP8749a-ev-AB) were tested in western blot (Fig. 1) and immunofluorescence studies (Fig. 2) on human osteosarcoma cells (U2OS) transfected with a human-MPV17 expression construct (Origene SC118652). The polyclonal antibody ab93374-as the only one yielding reproducible results-, revealed a band at the expected size of $20 \mathrm{kd}$ in western blot (Fig. 1 left panel) and a punctuate pattern in the immunofluorescence analyses (Fig. 2). When analysed for colocalisation with anti-complexIV mitochondrial subunit antibodies for mitochondrial and with anti-catalase antibodies for peroxisomal localisation, the Image J program revealed positive colocalisation for both. Remarkably, in both, western blot and immunofluorescence analyses, ab93374 reactivity was only detected in MPV17 expression construct transfected cells (Fig. 1 left panel, Fig. 2), while endogenous MPV17 protein was not traceable. Thus the ab93374 reaction was not very sensitive, because the endogenous MPV17 gene has been shown to be almost ubiquitously expressed at relatively high levels [25]. Furthermore, the apparent ambiguous localisation was unexpected, suggesting being potentially caused by transfection and overexpression artifacts. Therefore, mouse monoclonal antibodies raised against a recombinant Glutathion-S-Transferase (GST)-human MPV17 fusion protein, in which GST was fused to the $\mathrm{N}$-terminus of MPV17 [26], were studied. Two of them were reactive to the bacterially expressed fusion protein but not to GST alone [26].

These two, 6F5 and 5D2, were tested on Western blots of human U2OS cell extracts (Fig. 1, central panel). Both showed reactivity against a protein of the expected size

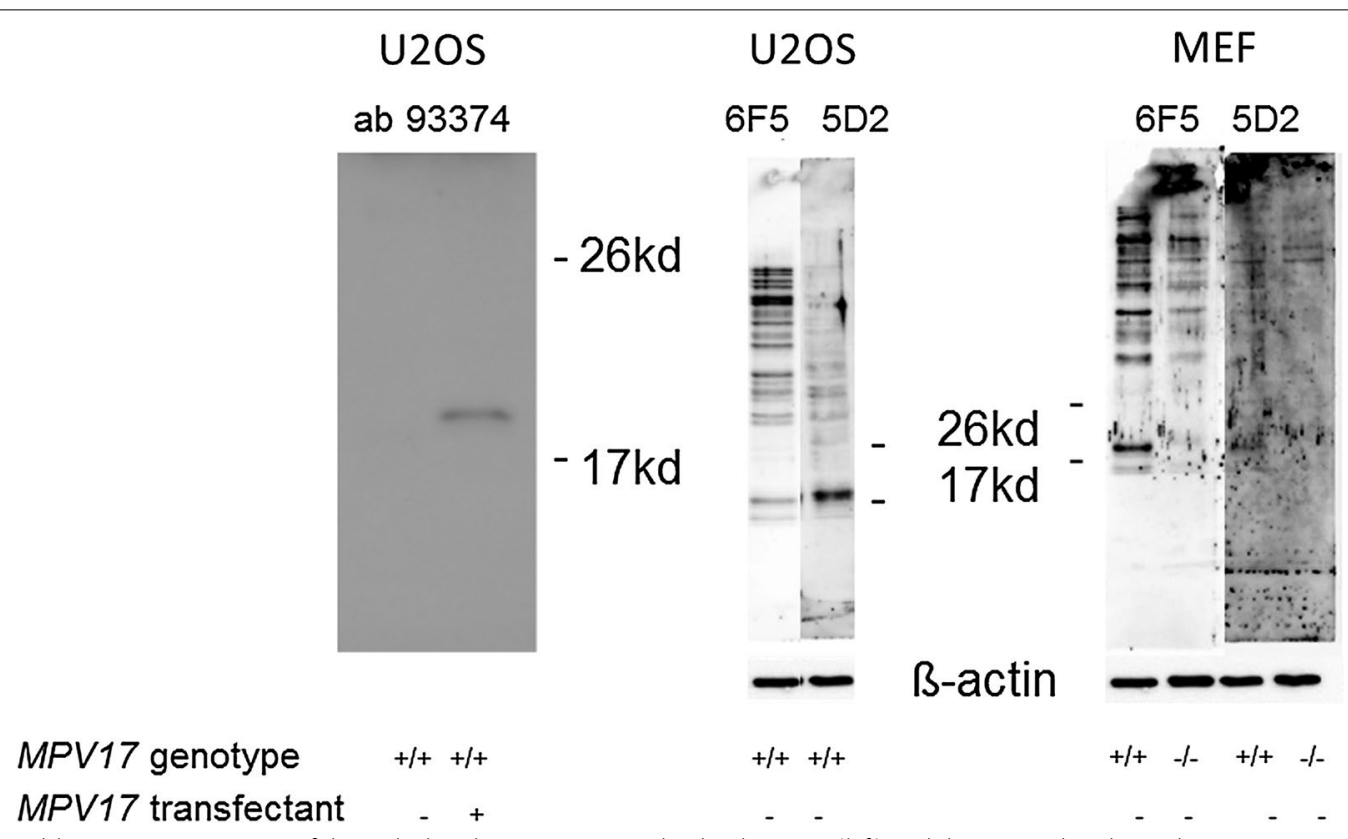

Fig. 1 Western blot immunoreactivity of the polyclonal anti-MPV17 antibody Ab 93374 (left) and the monoclonal anti- human MPV17 antibodies 6F5 and 5D2 on extracts of human and murine cells of different MPV17 genotypes. Left extracts from human U2OS cells, untransfected or transfected with the MPV17 expression clone SC118652 (origene), were probed with the antibody ab 93374 (abcam). Center untransfected U2OS cells with MPV17 +/+ endogenous genotype probed with the monoclonal antibodies 6F5 and 5D2. Right Murine embryo fibroblast (MEF) cells of MPV17 +/+ or MPV17-/- genotype probed with the monoclonal antibodies 6F5 and 5D2. Indicated marker sizes were transferred from Ponceau stained marker bands run in parallel. Loading was controlled as indicated by reaction of the filters with an anti-ß-actin antibody. $10 \mu \mathrm{g}$ of protein were separated on SDS gels and analysed by Western blot according to [16] (left panel), and Kinkley et al. [10] respectively 

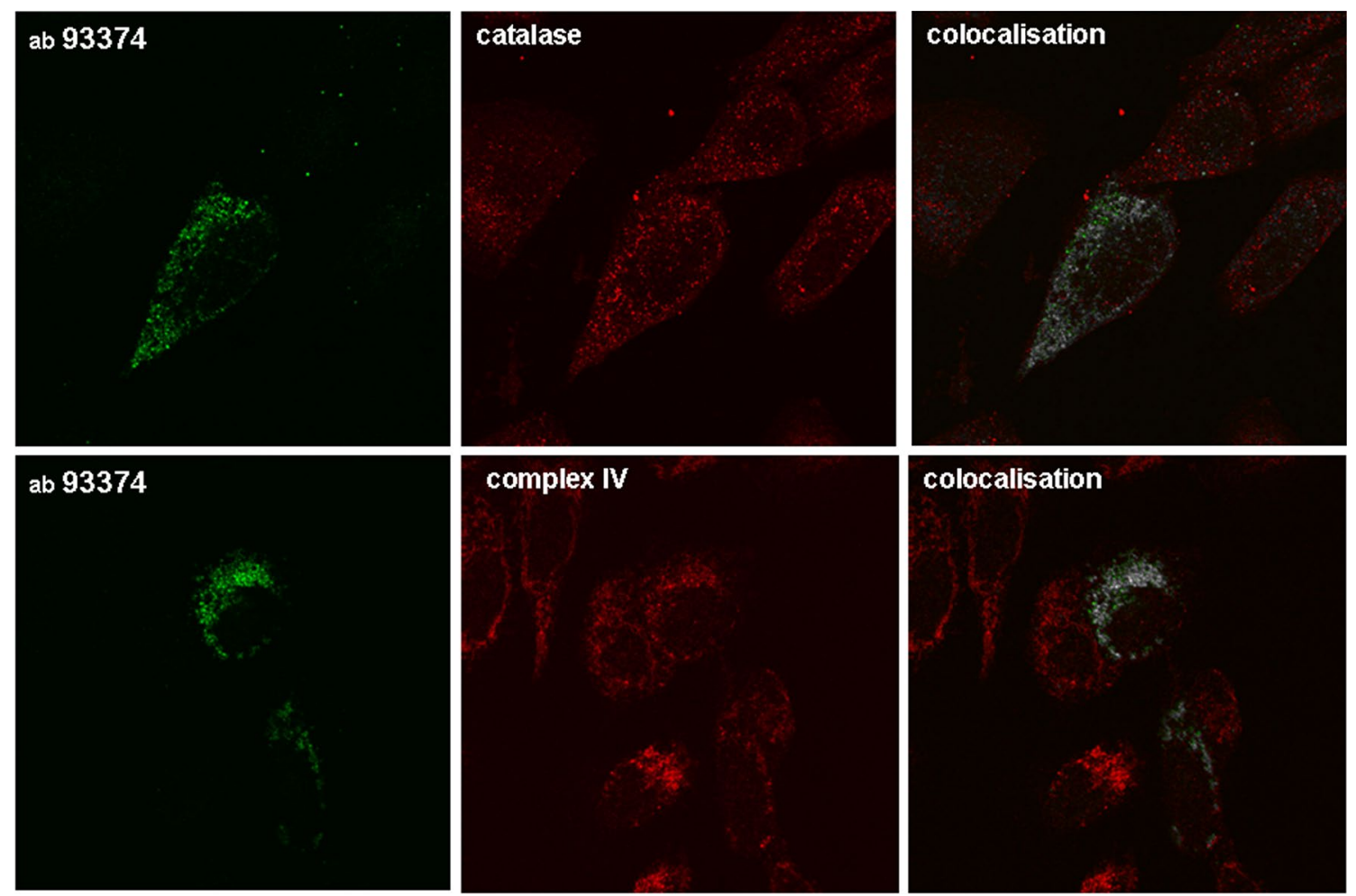

Fig. 2 Ab93374 (Abcam) recognizes MPV17 specific structures in U2OS cells transiently transfected with an MPV17 expression construct in immunofluorescence analysis. Ab93374 signal (green) is largely missing in nontransfected cells. The immunoreactivity does colocalise with peroxisomal (anti-catalase, mouse polyclonal, Abcam ab88650) and mitochondrial (anti-complex IV mitochondrial subunit I, mouse monoclonal, Invitrogen 459600) markers (red). Immunofluorescence (IF) method was according to Pircher et al. [16] using a MicroRadiance confocal scanning system (BioRad) in combination with a Zeiss Axiophot microscope. Colocalisation analysis was performed using the Fiji software of Image J and is illustrated by white dots

of approximately $20 \mathrm{kD}$. 6F5, however, recognised a number of additional bands evidencing cross-reactivity with other proteins. 5D2, by contrast, only detected a single band at $20 \mathrm{kD}$, the expected size. To investigate the specificity of these signals $M P V 17$ negative cells were necessary. For lack of such human cells from MDDS patients we tested these antibodies on extracts of mouse embryo fibroblasts (MEF) derived from $M P V 17+/+$ and MPV17 -/- cells from MPV17 knockout mice [26], respectively (Fig. 3, right panel). It revealed that the $20 \mathrm{kD}$ band recognised by $6 \mathrm{~F} 5$ was indeed the only one missing in MPV17 - / - cells, indicating that this band actually represented the MPV17 protein. 5D2, in contrast to the human cells, only showed a very weak signal on the mouse cells but this was again specific for the MPV17 positive genotype. Taken together, 6F5 recognised an epitope on the MPV17 protein, which this protein shares with a number of other proteins. On the other hand, 5D2 apparently displays exclusive anti-MPV17 reactivity, strong in human $\mathrm{U} 2 \mathrm{OS}$ cells but very weak in murine cells MPV17 positive and absent in MPV17 negative cells. This may indicate, that $5 \mathrm{D} 2$ is specific for a human specific epitope on the MPV17 protein, which is $92 \%$ identical between mouse and man [9]. Because the anti-human antibody was raised in mice, there might be a preference for such epitopes in the immune response.

When tested in immunofluorescence studies on U2OS cells, 6F5 did not display specific reactivity (data not shown), while 5D2 generated a characteristic punctate pattern, which in the Image J analysis of confocal microscopic pictures showed partial colocalisation with the peroxisomal marker PMP70 (polyclonal rabbit PMP70 antibody, gift from W. Just, University of Heidelberg), (Fig. 3 top). However, the pattern did not coincide with mitochondrial staining as displayed by MitoTracker Red (MP07510, Invitrogen) (Fig. 3 bottom). Thus, the staining pattern was not exclusively peroxisomal and not mitochondrial, as had been reported for MPV17 localisation in earlier studies, respectively $[21,26]$.

In search of other co-localising structures, experiments were performed with markers for other organels. As depicted in Fig. 4 we found partial colocalisation to a marker of the early endosomal compartment, EEA1 (Fig. 4 top), while there was no apparent co-localisation 

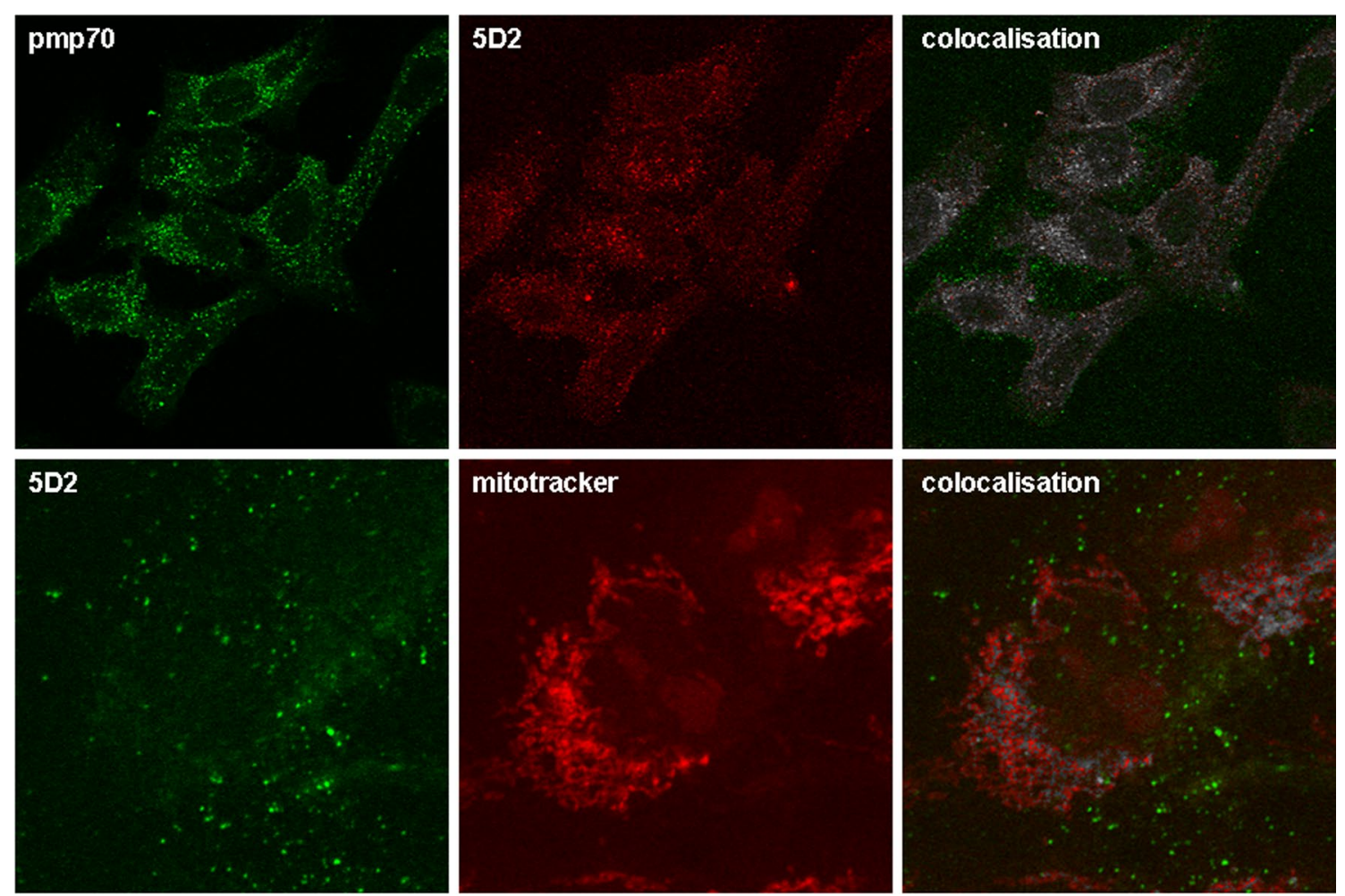

Fig. 3 In U2OS cells, 5D2 antibody detects a punctate pattern colocalising with a peroxisomal (PMP70) but not with a mitochondrial (MitoTracker) marker. Top Rabbit anti PMP70 antibody (decorated green) was a gift from W. Just, Heidelberg. Secondary antibodies: Alexa Fluor 555 anti-mouse and Alexa Fluor 488 anti-rabbit. Bottom Mitotracker 7510 (Invitrogen) was used as recommended by the supplier. Secondary antibody: Alexa 488 antimouse. IF method and microscopy according to Kinkley et al. [10] using a Zeiss LSM confocal microscope. Mathematical colocalisation analysis was performed using the Fiji software of Image $J$ and is illustrated by white dots

with RAB7, a marker of the late endosomal compartment (data not shown). In addition, clear colocalision was detected with Cathepsin D (not shown) and LAMP 1 (Fig. 4 bottom). Figure 5 shows a single cell analysis of LAMP1-5D2 costaining, in which in addition the optical overlay colocalisation was added to the mathematical colocalisation analysis by the Fiji software of the ImageJ program package. Thus, 5D2 recognizes a structure that is present in endosomes and lysosomes in addition to peroxisomes. This in marked contrast to earlier localisations of MPV17 with different antibodies.

\section{Conclusions}

In this work we have characterised two monoclonal antibodies raised against a bacterially expressed $\mathrm{N}$-terminal GST fused to the human MPV17 protein. These antibodies, specific for the MPV17 part of the fusion protein recognise a band of the expected size in human U2OS cells. While 6F5 cross reacts with other proteins 5D2 appears to be monospecific. 5D2 creates a punctate pattern in immunofluorescence studies partially colocalising with peroxisomal, early endosomal, and lysosomal markers but not with mitochondria. 5D2 does bind only weakly but specifically to the murine MPV17 homolog (Fig. 1), suggesting that the mouse monoclonal might bind to an epitope where murine and human MPV17 diverge. This could be the c-terminus of the molecule, which is the region of strongest divergence. In line with this idea is the fact that the c-terminus of the MPV17 molecule was exposed in the bacterial n-terminal fusion to GST antigen used for immunisation. Moreover, MPV17 molecules fused at the c-terminus to other proteins cannot be detected by 5D2 (data not shown).

The commercial anti-human MPV17 antibody used in our first approach was a polyclonal rabbit antibody raised against GST-MPV17 fusion protein (abcam ab 93374) and has produced erroneous or ambiguous results (Fig. 2). The rabbit polyclonal antibody from Proteintech used by [21] leading to mitochondrial localisation was raised against the identical GST-MPV17 fusion and might have been error-prone as well. Furthermore, in addition to possible transfection artifacts, MPV17 and 

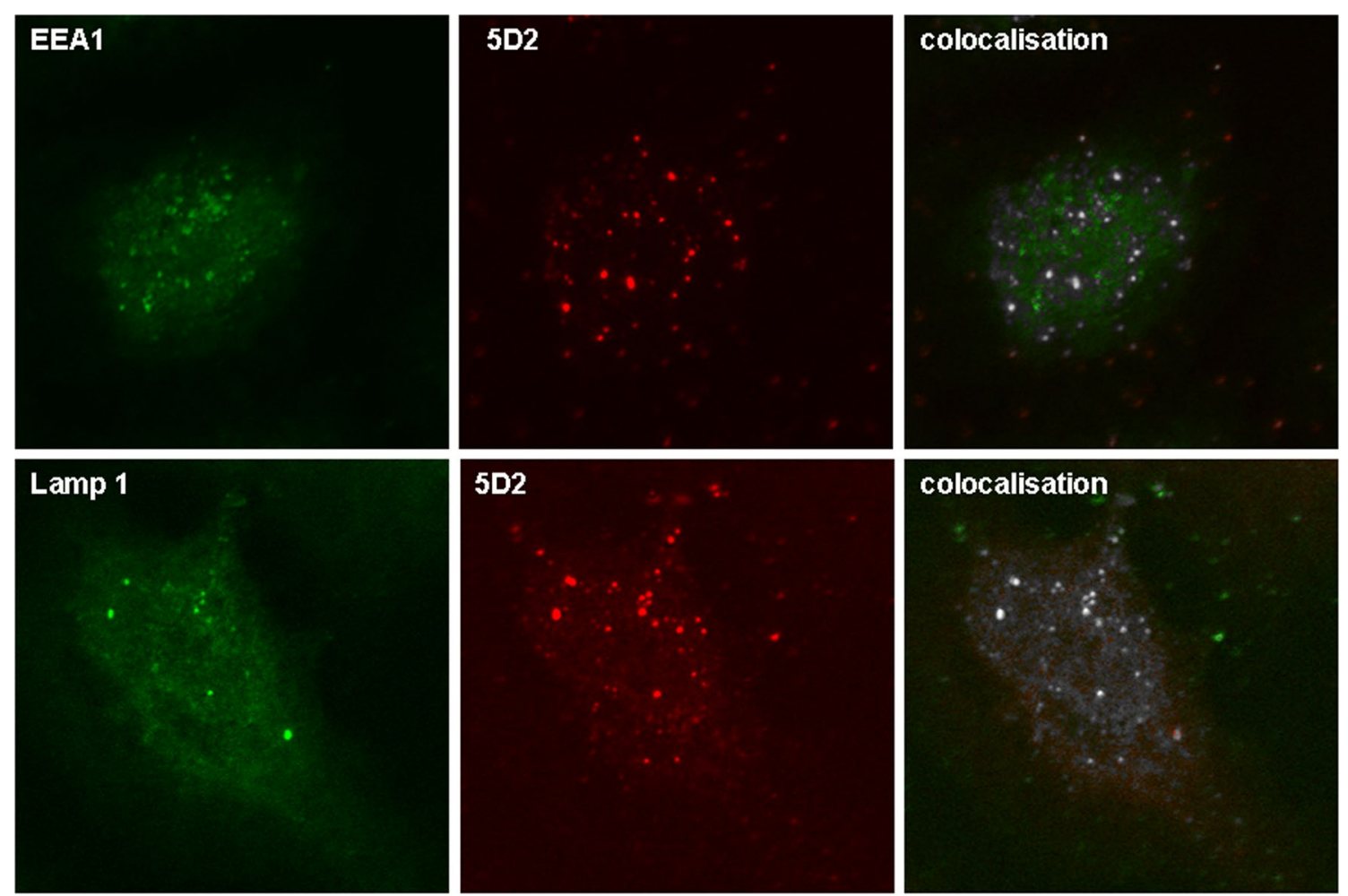

Fig. 4 5D2 colocalisation with a marker of early endosomes (EEA1) and lysosomes (LAMP1) in U2OS cells. Top Partial colocalisation of 5D2 with EEA1 (rabbit anti-human). Bottom colocalisation of 5D2 with LAMP1 in U2OS cells. The IF method, secondary antibodies, and Image J analysis were used as described in the legend of Fig. 3
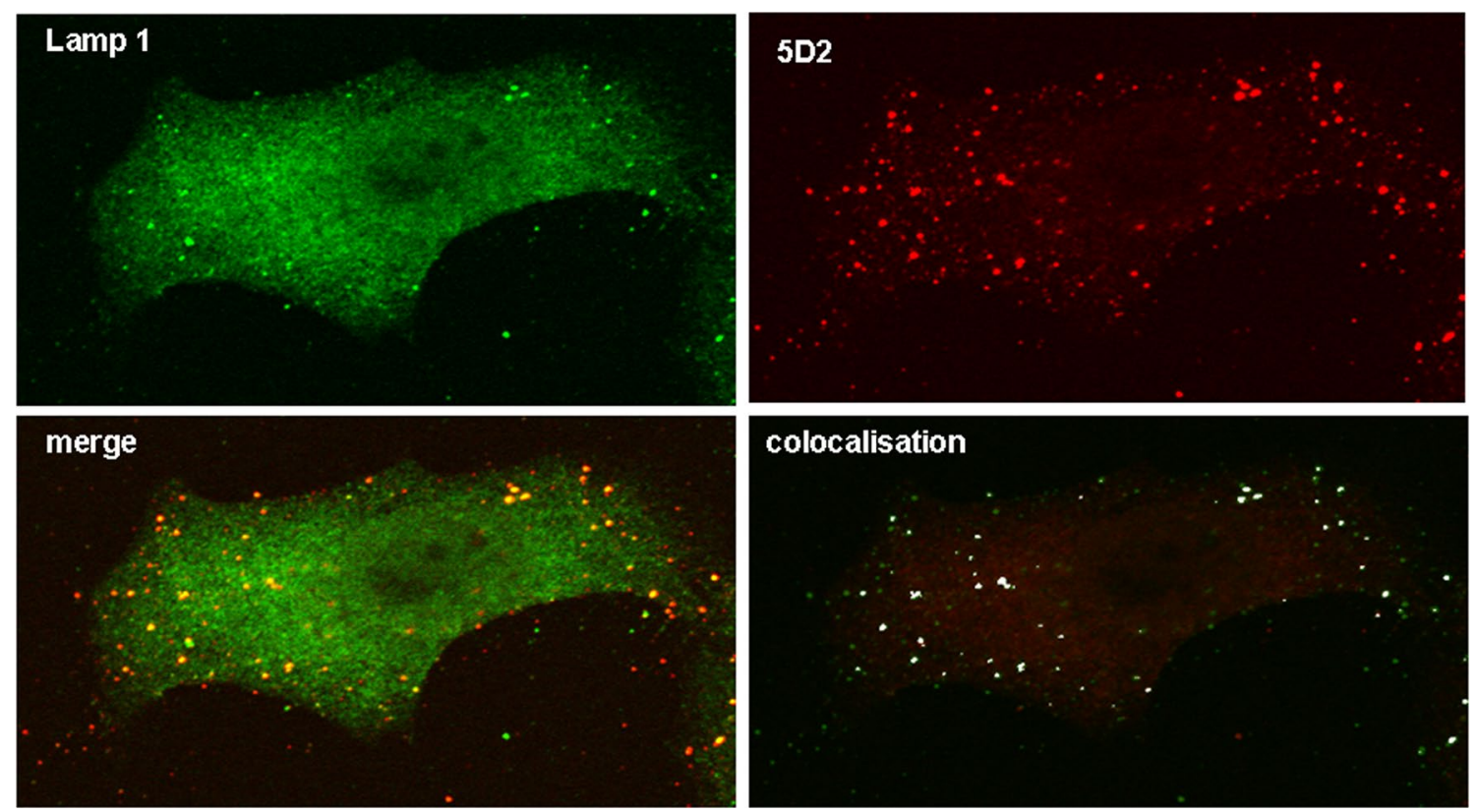

Fig. 5 Colocalisation of 5D2 and LAMP1 mediated immunofluorescence in a U2OS single cell. IF and analysis of 5D2 and Colocalisation of 5D2 with LAMP1 in U2OS cells. The IF method, secondary antibodies, and Image J analysis were used as described in the legend of Fig. 3. In addition a merge of the two immunofluorescence pictures is depicted 
related proteins such as PXMP22 may localise erroneously, when they are fused to detection tags (unpublished and [3].

MPV17 deficiencies in humans can cause fatal liver disease mediated by mitochondrial DNA depletion in liver cells [21]. However, primary human liver cells were not available for our studies. We therefore had to perform this study on unrelated human tumour cells, and it is possible, that the MPV17 protein might localise differently in different cell types. Yet, preliminary studies on human primary skin fibroblasts show MPV17 colocalisation with LAMP1 as well (unpublished results) and thus corroborate the data on U2OS cells presented above. Thus, our data raise the question of how the mitochondrial MDDS phenotype is generated if the MPV17 gene product is found mainly in other organelles. It has been shown that MPV17 protein forms a membrane channel with a diameter allowing low molecular weight molecules to pass [1] but the role of the channel is still elusive, particularly considering the different phenotypes that mutations of it can cause in different species [13]. We look forward to provide the antibodies described here to approach these open questions.

\begin{abstract}
Authors' contributions
HW: Making of constructs, western blots, immunohistochemistry, confocal microscopy. HP and PJD: contribution to the Westernblot in Fig. 1, transfection and immunohistochemistry depicted in Fig. 2. SH and PK: help with growth and purification monoclonal antibodies. SG, MV and JKH: help with and the production of MPV17 negative MEF cells. RMZ: generation of monoclonal antibodies. ES: help with confocal microscopy and Fiji software. KR and HW: technical and financial support; critical reading of the manuscript and most valuable discussion. All authors read and approved the final manuscript.
\end{abstract}

\section{Author details \\ ${ }^{1}$ Heinrich-Pette-Institute, Leibniz-Institute for Experimental Virology, Martinistrasse 52, 20251 Hamburg, Germany. ${ }^{2}$ Bonn-Rhein-Sieg University, von Liebig Strasse 20, 53359 Rheinbach, Germany. ${ }^{3}$ Institute for Biomedical Aging Research, University of Innsbruck, Rennweg 10, 6020 Innsbruck, Austria. ${ }^{4}$ Institutes of Molecular Medicine and Experimental Immunology, Universität Bonn, 53105 Bonn, Germany. ${ }^{5}$ Department of Biochemistry, Biocenter Oulu, University of Oulu, Fl-90014 Oulu, Finland. ${ }^{6}$ School of Biological Sciences, University of Essex, Colchester CO4 3SQ, UK. ${ }^{7}$ Max Planck Institute for Plant Breeding Research, Carl-von-Linné-Weg 10, 50829 Cologne, Germany.}

\section{Acknowledgements}

We thank Dr. Alexander Bartelt and Dr. Katrin Kollmann, UKE Hamburg, for marker antibodies and valuable discussions. This work was supported by the Heinrich-Pette-Institute, Hamburg, Germany; the Institute for Biomedical Aging Research, University of Innsbruck, Austria; the Institutes of Molecular Medicine and Experimental Immunology, Universität Bonn, Germany; and the Department of Biochemistry, Biocenter, University of Oulu, Finland.

\section{Competing interests}

The authors declare that they have no competing interests.

\section{Ethics}

All work concerned tissue culture cells for which no specific licences were required. For isolation of the mouse embryonic fibroblasts the use of experimental animals was conducted under the permission of the Animal Experimentation Board of Finland (ESLH-2009-04840/Ym-23).
Received: 17 July 2015 Accepted: 16 February 2016

Published online: 27 February 2016

\section{References}

1. Antonenkov VD, Isomursu A, Mennerich D, Vapola MH, Weiher $\mathrm{H}$, Kietzmann T, Hiltunen JK. The human mitochondrial DNA depletion syndrome gene MPV17 encodes a non-selective channel that modulates membrane potential. J Biol Chem. 2015;290:3840-61.

2. Binder CJ, Weiher H, Exner M, Kerjaschki D. Glomerular overproduction of oxygen radicals in MPV17 gene-inactivated mice causes podocyte foot process flattening and proteinuria: a model of steroid-resistant nephrosis sensitive to radical scavenger therapy. Am J Pathol. 1999;154:1067-75.

3. Brosius U, Dehmel T, Gärtner J. Two different targeting signals direct human peroxisomal membrane protein 22 to peroxisomes. J Biol Chem. 2002;277:774-84.

4. Copeland WC. Defects in mitochondrial DNA replication and human disease. Crit Rev Biochem Mol Biol. 2012;47:64-74.

5. El-Hattab AW, Li F-Y, Schmitt E, Zhang S, Craigen WJ, Wong L-JC. MPV17associated hepatocerebral mitochondrial DNA depletion syndrome: new patients and novel mutations. Mol Genet Metab. 2010;99:300-8.

6. Iida R, Yasuda T, Tsubota E, Takatsuka H, Matsuki T, Kishi K. Human MPV17like protein is localized in peroxisomes and regulates expression of antioxidant enzymes. Biochem Biophys Res Commun. 2006;344:948-54.

7. Kaldi K, Diestelkötter P, Stenbeck G, Auerbach S, Jäkle U, Mägert HJ, Wieland FT, Just WW. Membrane topology of the $22 \mathrm{kDa}$ integral peroxisomal membrane protein. FEBS Lett. 1993;315:217-22.

8. Karadimas CL, Vu TH, Holve SA, Chronopoulou P, Quinzii C, Johnsen SD, Kurth J, Eggers E, Palenzuela L, Tanji K, Bonilla E, De Vivo DC, DiMauro S, Hirano M. Navajo neurohepatopathy is caused by a mutation in the MPV17 gene. Am J Hum Genet. 2006;79:544-8.

9. Karasawa M, Zwacka RM, Reuter A, Fink T, Hsieh CL, Lichter P, Francke U, Weiher $\mathrm{H}$. The human homolog of the glomerulosclerosis gene MPV17: structure and genomic organization. Hum Mol Genet. 1993;2:1829-34.

10. Kinkley S, Staege H, Mohrmann G, Rohaly G, Schaub T, Kremmer E, Winterpacht A, Will H. SPOC1: a novel PHD-containing protein modulating chromatin structure and mitotic chromosome condensation. J Cell Sci. 2009;122:2946-56.

11. Meyer zum Gottesberge AM, Reuter A, Weiher H. Inner ear defect similar to alport's syndrome in the glomerulosclerosis mouse model MPV17. Eur Arch Otorhinolaryngol. 1996;253:470-4.

12. Krauss J, Astrinides P, Frohnhöfer HG, Walderich B, Nüsslein-Volhard C. Transparent, a gene affecting stripe formation in Zebrafish, encodes the mitochondrial protein MPV17 that is required for iridophore survival. Biology Open. 2013;2:703-10.

13. Löllgen $\mathrm{S}$, Weiher $\mathrm{H}$. The role of the MPV17 protein mutations of which cause mitochondrial DNA depletion syndrome (MDDS): lessons from homologs in different species. Biol Chem. 2015;396:13-25. doi:10.1515/ hsz-2014-0198.

14. O'Bryan T, Weiher H, Rennke HG, Kren S, Hostetter TH. Course of renal injury in the MPV17-deficient transgenic mouse. J Am Soc Nephrol. 2000;11:1067-74

15. Papeta N, Zheng Z, Schon EA, Brosel S, Altintas MM, Nasr SH, Reiser J, D'Agati VD, Gharavi AG. Prkdc participates in mitochondrial genome maintenance and prevents adriamycin-induced nephropathy in mice. J Clin Invest. 2010;120:4055-64.

16. Pircher H, Straganz GD, Ehehalt D, Morrow G, Tanguay RM, Jansen-Dürr P. Identification of human fumarylacetoacetate hydrolase domain-containing protein 1 (FAHD1) as a novel mitochondrial acylpyruvase. J Biol Chem. 2011;286:36500-8.

17. Reinhold R, Krüger V, Meinecke M, Schulz C, Schmidt B, Grunau SD, Guiard B, Wiedemann N, van der Laan M, Wagner R, Rehling P, Dudek J. The channel-forming SYM1 protein is transported by the TIM23 complex in a presequence-independent manner. Mol Cell Biol. 2012;32:5009-21.

18. Rokka A, Antonenkov VD, Soininen $\mathrm{R}$, Immonen $\mathrm{HL}$, Pirilä $\mathrm{PL}$, Bergmann $\mathrm{U}$, Sormunen RT, Weckström M, Benz R, Hiltunen JK. Pxmp2 is a channelforming protein in mammalian peroxisomal membrane. PLoS One. 2009;4:e5090 
19. Rosa ID, Durigon R, Pearce SF, Rorbach J, Hirst EM, Vidoni S, Reyes A, BreaCalvo G, Minczuk M, Woellhaf MW, Herrmann JM, Huynen MA, Holt IJ, Spinazzola A. MPV17L2 is required for ribosome assembly in mitochondria. Nucleic Acids Res. 2014;42:8500-15.

20. Schenkel J, Zwacka RM, Rutenberg C, Reuter A, Waldherr R, Weiher $\mathrm{H}$. Functional rescue of the glomerulosclerosis phenotype in MPV17 mice by transgenesis with the human MPV17 homologue. Kidney Int. 1995:48(1):80-4

21. Spinazzola A, Viscomi C, Fernandez-Vizarra E, Carrara F, D'Adamo P, Calvo S, Marsano RM, Donnini C, Weiher H, Strisciuglio P, Parini R, Sarzi E, Chan A, DiMauro S, Rötig A, Gasparini P, Ferrero I, Mootha VK, Tiranti V, Zeviani M. MPV17 encodes an inner mitochondrial membrane protein and is mutated in infantile hepatic mitochondrial DNA depletion. Nat Genet. 2006;38:570-5.

22. Trott A, Morano KA. SYM1 is the stress-induced saccharomyces cerevisiae ortholog of the mammalian kidney disease gene MPV17 and is required for ethanol metabolism and tolerance during heat shock. Eukaryot Cell. 2004;3:620-31.
23. Uusimaa J, Evans J, Smith C, Butterworth A, Craig K, Ashley N, Liao C, Carver J, Diot A, Macleod L, Hargreaves I, Al-Hussaini A, Faqeih E, Asery A, Al Balwi M, Eyaid W, Al-Sunaid A, Kelly D, van Mourik I, Ball S, Jarvis J, Mulay A, Hadzic N, Samyn M, Baker A, Rahman S, Stewart H, Morris AAM, Seller A, Fratter C, Taylor RW, Poulton J. Clinical, biochemical, cellular and molecular characterization of mitochondrial DNA depletion syndrome due to novel mutations in the MPV17 gene. Eur J Hum Genet. 2013;22(2):184-91.

24. Viscomi C, Spinazzola A, Maggioni M, Fernandez-Vizarra E, Massa V, Pagano C, Vettor R, Mora M, Zeviani M. Early-onset liver mtDNA depletion and late-onset proteinuric nephropathy in MPV17 knockout mice. Hum Mol Genet. 2009;18:12-26.

25. Weiher H, Noda T, Gray DA, Sharpe AH, Jaenisch R. Transgenic mouse model of kidney disease: insertional inactivation of ubiquitously expressed gene leads to nephrotic syndrome. Cell. 1990;62:425-34.

26. Zwacka RM, Reuter A, Pfaff E, Moll J, Gorgas K, Karasawa M, Weiher H. The glomerulosclerosis gene MPV17 encodes a peroxisomal protein producing reactive oxygen species. EMBO J. 1994;13:5129-34.

\section{Submit your next manuscript to BioMed Central and we will help you at every step:}

- We accept pre-submission inquiries

- Our selector tool helps you to find the most relevant journal

- We provide round the clock customer support

- Convenient online submission

- Thorough peer review

- Inclusion in PubMed and all major indexing services

- Maximum visibility for your research

Submit your manuscript at www.biomedcentral.com/submit
(O) BioMed Central 